\title{
Evaluation of ON-OFF Schemes and Linear Prediction Methods for Increasing Energy Efficiency in Mobile Broadband Networks
}

\author{
Dario Sabella ${ }^{1}$, Marco Caretti ${ }^{1}$, William Tomaselli ${ }^{1}$, Valerio Palestini ${ }^{1}$, \\ Bruno Cendón ${ }^{2}$, Javier Valino ${ }^{2}$, Arturo Medela ${ }^{2}$, \\ Yolanda Fernández ${ }^{2}$, and Luis Sanchez ${ }^{3}$ \\ ${ }^{1}$ Telecom Italia, via Guglielmo Reiss Romoli, 274 - Turin, Italy \\ \{dario.sabella, marco.caretti, guglielmo.tomaselli, \\ valerio.palestini\} atelecomitalia.it \\ 2 TTI Norte, Avda. Albert Einstein 14, 39010, Santander, Spain \\ \{bcendon, jvalino, amedela\} @tst-sistemas.es, \\ yfernandez@ttinorte.es \\ ${ }^{3}$ Universidad de Cantabria, Plaza de la Ciencia s/n, 39005, Santander, Spain \\ lsanchez@tlmat.unican.es
}

\begin{abstract}
Nowadays, energy efficiency has become a major issue in mobile networks operation. Due to the exponential rise in the number of wireless Internet-connected mobile devices reducing electrical energy consumption is not only a matter of showing environmental responsibility, but also of substantially reducing their operational expenditure. However, energy reduction cannot be pursued at any cost and appropriate service has to be supported. Among the diverse hardware and software solutions available, this paper focuses on the dynamic operation of cellular base stations, in which redundant base stations are switched off during periods of low traffic. Besides, we are also describing the use of prediction mechanisms in order to make a proper decision on when to take that action. The proposed schemes are assessed by means simulations using both theoretical and real load models.
\end{abstract}

Keywords: Green radio, energy saving, ON/OFF scheme, linear predictors, mobile communications, 3G, LTE, eNodeB, traffic load curves.

\section{Introduction}

The exponential growth in the number of devices connected to wireless networks makes the power consumption associated with their use also increase, which is against energy saving policies in place today. However, from the perspective of cellular network operators, reducing electrical energy consumption is not only a matter of being "green" and responsible, it is also very much an economically important issue: in fact, a significant portion of the operational expenditure (OPEX) of a cellular network goes to pay the electricity bill. From [1][2], it can be estimated that the mobile network OPEX for electricity globally is more than $\$ 10$ billion dollars today. The key source 
of energy usage in cellular networks is the operation of Base Station (BS) equipment. It has been estimated that BSs contribute to $60-80 \%$ of the total energy consumption [3]. Energy efficiency with respect to BSs has been considered in all stages of cellular networks, including hardware design and manufacture, deployment, and operation.

In this paper we will focus at the access network management level. So far, the different studies in the field of the switching on and off of BSs have focused on estimating the savings that could be achieved by dynamically adapting the operation of cellular network infrastructure to the required load. Initially load behaviour was simulated using linear, Gaussian or averaged models [3] [5]. In all of them the curves consists in monotonically decreasing or increasing functions and thus only a single low load "valley" appears. More recently, traffic profiles retrieved from a real network have been used for the same assessment [4], but still only the estimated savings are presented. The proposed scheme tries to dynamically minimize the number of active BSs to meet the traffic variation in the network, assuming that remaining active BSs are able to cope with the traffic that active users are generating. The optimum solution would require constantly mimicking the behaviour of the load profile and switching on and off cells as the traffic respectively increases or decreases. Since this way of operation demands complex self-organization issues, a simple scheme is designed, defining a priori the number of BSs that shall be put into idle mode, bearing in mind that the remaining sites could provide the required coverage to that specific area.

\section{Reference Scenario}

The main aim of the work presented in this paper is to create an intelligent network management mechanism to switch on and off BSs depending on the traffic load, reshaping at the same time the cell topology, always maintaining Quality of Service (QoS) and Quality of Experience (QoE), so the user will not notice any disruption in the service. Regarding cell geometry and positioning, the studies will consider typical grid with hexagonal geometry as it is the preferred and most conventional configuration. It is also important to clarify that tri-sectorial antennas will be used, in opposition to the omni-directional ones that were implemented in the previous studies, thus achieving results closer to reality. This option has been selected according to the reference scenarios defined in [6], where a distinction is made among three of them: urban, suburban and rural ones. All of them assume a homogeneous hexagonal deployment and uniform user distribution in order to carry out the different tests. Based on this premise, and taking into account those real networks constraints, the choice is to focus the work on two specific scenarios. In the first one of them, when the load decreases 3 out of 4 cells can be switched off, so just 1 remains active and a big amount of power is saved. This way, the Inter Site Distance (ISD) increases to the double of the size it had before the algorithm goes into action. Meanwhile, the alternative scheme presents the switching off of 8 out of 9 cells, increasing the ISD by a factor of 3. These two options can be seen in Fig. 1. 


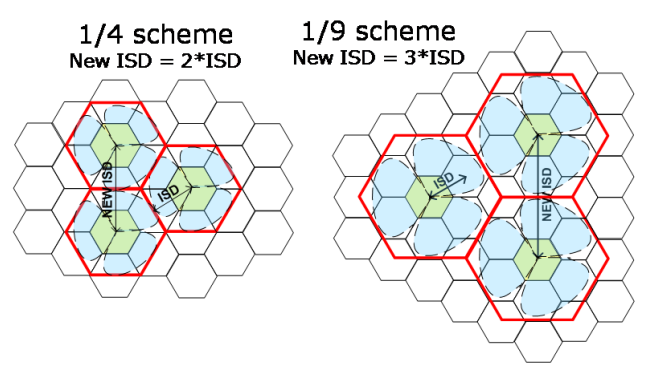

Fig. 1. 1/4 and $1 / 9$ schemes

The deployment scenarios selected for the evaluation of the proposed solution derive from several simulations, extracted from [7] and used to help the authors to decide when and how to switch between different ISD scenarios (see Fig. 2, derived for simulations conducted within the EARTH project). Moreover, the SOTA model for power consumption, presented in Fig. 3, will be used to calculate the power transmitted in each case. By introducing instantaneous load of the BS (for instance, serving $10 \mathrm{Mbps}$ when the maximum capacity is $50 \mathrm{Mbps}$ will mean a $20 \%$ load), it is possible to directly obtain consumption in terms of Watts.

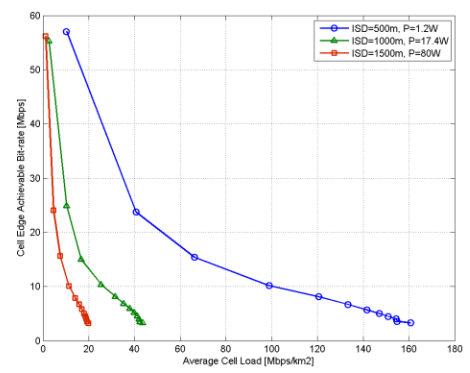

Fig. 2. Average cell load depending on the ISD and the Power transmitted

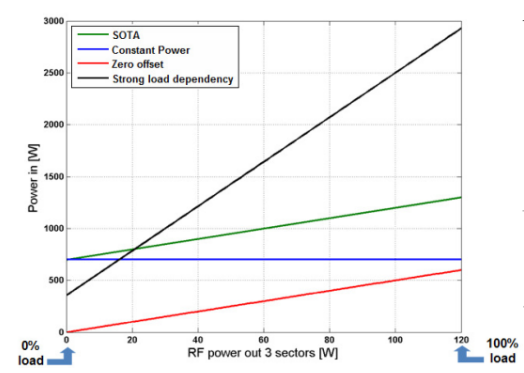

Fig. 3. Linear power models [7]

As regards reference system and main simulation parameters (described in [7]), a high traffic scenario (with peak traffic equal to $120 \mathrm{Mbps} / \mathrm{Km}^{2}$ ) will be chosen as input, considering a macro BS with special capabilities to modify their transmitted power when changing the scenario (using MACRO BS able to reduce their power consumption when changing from $1 / 9$ to $1 / 4$ or $1 / 1$ and not the other way around, because using MICRO BS enhancing their power would translate into big consumptions for $1 / 4$ or $1 / 9$ scenarios), and also taking in consideration 3 different ISDs $(0.5,1$ and $1.5 \mathrm{Km})$ between cells that will derive in different cell load curves. In addition, a cubic interpolation of data will be using the power model for a Macro BS but making some corrections adapting it to tri-sectorial antennas, following the formula shown below.

$$
\mathrm{P}_{\mathrm{OUT}}=720+5 \cdot\left(3 \cdot \mathrm{P}_{\mathrm{IN}}\right) \mathrm{P}_{\mathrm{OUT}}=720+5 \cdot\left(3 \cdot \mathrm{P}_{\mathrm{IN}}\right)
$$


In this last formula, 720 factor corresponds to input power (due to physical components on the $\mathrm{BS}$ ), and the 3 factor multiplying $\mathrm{P}_{\mathrm{IN}}$ (introduced as \% load) responds to the tri-sector scheme.

\subsection{Methodology and Data Retrieval from a Real Mobile Network}

In order to study the performance of the ON-OFF scheme in a real network, the operation of the algorithm when it is fed with real traffic curves has been tested. In particular, a set of data traffic profiles was taken from Telecom Italia mobile network: the dataset, extracted by a TIM mobile network monitoring system with a fixed time resolution, is relative to an RBS currently running in the Telecom Italia mobile network. In particular, for each sector, data samples automatically provided by the monitoring system represent the average values of a specific KPI over 15 minutes time intervals (e.g. for data bandwidth the sample represents the amount of data transferred in 15 minutes), and then a daily profile is represented by 96 consecutive values. Each sample is also averaged over the 5 working days of the week, in order to filter effects due to spurious data peaks and increase the reliability of the provided profile.

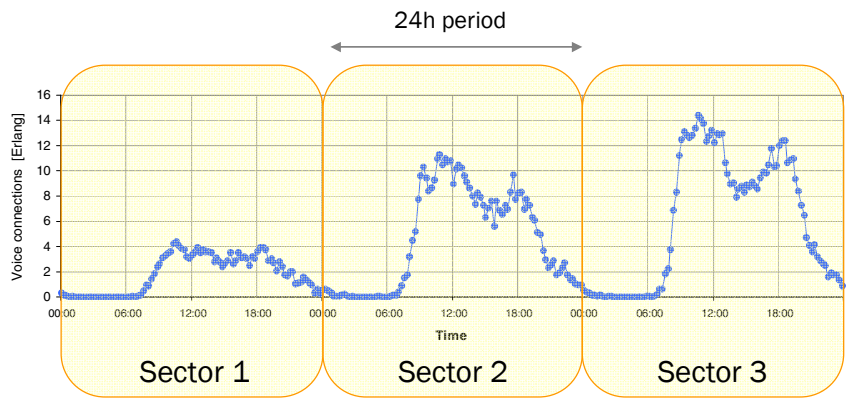

Fig. 4. Example of voice connections for UMTS sectors

Fig. 4 shows an example of the data profiles used, relative to a RBS installed and running in the mobile network. The site considered to retrieve this data profile is a 3sectorial macro site located in an urban environment, equipped with GSM 900, GSM 1800 , UMTS and HSDPA carriers, and captures all operator's traffic demand in the covered area (2G, $3 \mathrm{G}$ and $3.5 \mathrm{G})$. For easing the visualization, the 3 sectors are depicted in the same plot. The trend of extracted data profiles showed a typical daily oscillation of the traffic, consistent with average profiles contained in [6][7], but with the important difference that while literature curves are averaged over an entire network, data profiles used in this paper are relative to single sites, in order to better show particular burst effects of the traffic variation. The resulting curves are then suitable for a fine tuning of the developed prediction algorithms, and more reliable to give a performance indication of the ON-OFF schemes in a real mobile network. 


\section{Prediction Methods}

The appearance of several valleys during a regular day load imposes the introduction of additional mechanisms to improve the performance of the ON-OFF scheme. An important upgrade consists on combining instantaneous and historical data to take the most appropriate decision at any time. To do so, it is mandatory to gather the instantaneous data load together with a window of previous load conditions. The next step should be to combine this data with a predictor using coefficients historically tuned in order to determine the threshold of considering or not a short period.

Based on different daily load curves models, the evaluation started using diverse ON-OFF schemes: first static ones have been analyzed, and then the possibility of combining them dynamically has also been evaluated. So far, the difference lies in the number of ON-OFF schemes applied in the period of one day, this means from $t=0$ to $\mathrm{t}=\mathrm{T}=24$ hours; while the static model applies only one ON-OFF scheme a day, the dynamic model allows several transitions depending on the load. The upper side of Fig. 5 presents a comparison between power savings reduction using static and dynamic ON-OFF schemes applied on a simplified linear traffic model illustrated on the lower side of Fig. 5. In this case, two static ON-OFF schemes, 1/2 and 1/4 are compared with a dynamic model that combines both schemes. The static model only takes into account one specific threshold (either $1 / 2$ or $1 / 4$ ), whereas the dynamic model switches between both thresholds, applying the most appropriate in each situation. For the considered ON-OFF schemes, 1/x denotes that only 1 out of $\mathrm{x}$ base stations remain in $\mathrm{ON}$ state after the OFF management action.

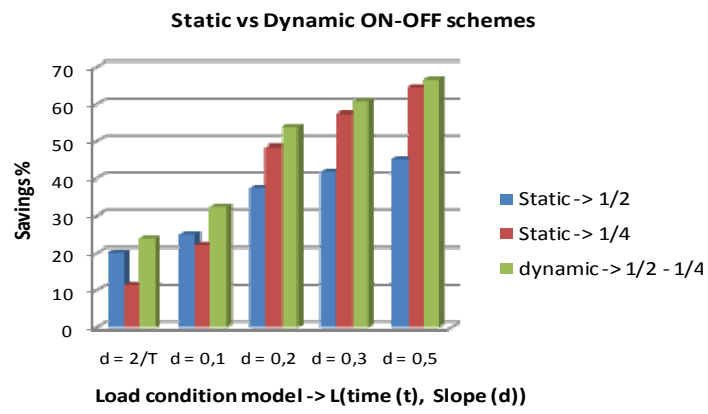

Fig. 5. Comparison between static and dynamic ON-OFF schemes in case of simplified linear traffic models

After seeing the theoretical energy savings in a simplified linear world, the next step is evaluating what potentials are in real networks [6][8]. Fig. 6 illustrates the energy saving potential in real life measurements provided by EARTH project partners. In the figure different static schemes are compared assuming a uniform omni-cell deployment where the daily variation of the traffic is according to real measurements. It can be concluded that more than $25 \%$ of the energy could be saved in an omni-cell deployment scenario. 


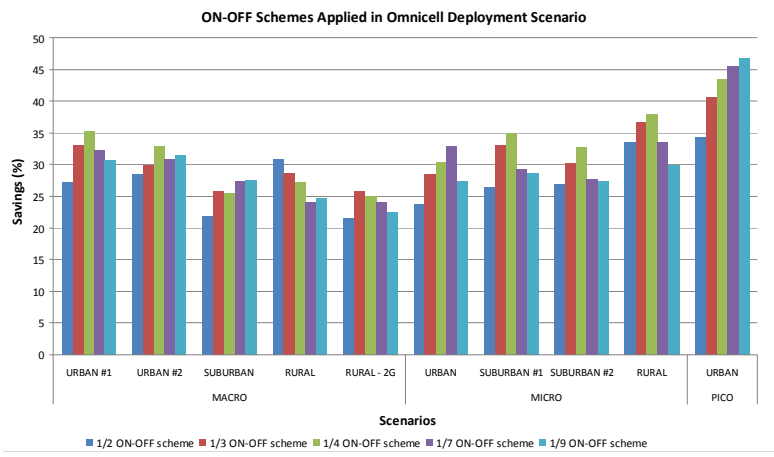

Fig. 6. ON-OFF schemes applied in omnicell deployment scenario

However, in contrast to the above linear model, the cell level traffic variation is substantially much more complex. Thus, other important aspect to be considered when applying the ON-OFF scheme is the necessity to limit the situations in which it is safe and helpful to apply this scheme. There might be situations in which several time slots in which it might be possible to apply an ON-OFF scheme exist. While some of them could actually be used, on others initiating the BS shut down might be counterproductive if the slot is not wide enough. For this purpose it is therefore essential to be aware of two critical issues: the time it takes for a BS to change state (from on to off or vice versa) and the expected value of the load on the cell during this time.

For the first one, a sensible assumption is to have slots larger than 30 minutes to have an efficient application of the algorithm, thus estimating conservatives on and off times. Pertaining to the network load on those future 30 minutes, some kind of predictor is needed in order to be able to foresee the future trends of the data curve. Therefore, in order to reach the pursued goals and avoid an inefficient use of the ON-OFF scheme, the utilization of some studies based on linear prediction will help. These kinds of predictors can tell whether the load curve is going to decrease (so the ON-OFF schemes can be applied) or it is going to increase after reaching some threshold, which would lead to a bad use of ON-OFF schemes. Initially the algorithms have been used mostly on voice call profiles (as data call profiles are very bursty), but trying to make a better approach the path taken has consisted in averaging real data curves. Such linear predictors are characterized by the following parameters:

- Model: Curve taken as reference to make predictions. In a system capable of learning, this model can change over time based on averages of the measures obtained daily. Similarly, several models that apply in different situations (weekdays, holidays...) can be used. In the case of ON-OFF algorithm, this model will be an averaged load curve. From here, an array of coefficients, which serve to weigh the actual data, is obtained.

- Window: Number of samples that are intended to predict from the actual data and model. The larger the window, the more errors will be committed, because we have to consider when making the prediction of all samples (except the first) entry must be entered as previous predictions, which are not accurate. 
- Predictor order: Number of coefficients or measures taken as a reference from the model curve to construct the coefficient matrix. Higher orders cause predictions tightly adjusted to reality, but at the expense of increased processing times.

\section{Power Savings}

Applying all the previously mentioned inputs together (Averaged/Ideal data curve, High density urban scenario, BS power assumptions, linear predictors with $60^{\text {th }}$ order and 30 minutes window), and evaluating a 24 hour period, it is possible to obtain theoretical savings, corresponding to ideal and smooth behaviour of data traffic. The primary savings are obtained when switching from $1 / 1$ to $1 / 4$ scheme. Additional ones are observed during night time, when it is possible to switch from this $1 / 4$ scheme to 1/9. The total savings are $795.47 \mathrm{~W} / \mathrm{Km}^{2}$ in a period of 7 hours and 1 minute. It means that, on average, BS on our theoretical scenario, are wasting energy during 7 out of the 24 hours of the day.

Apart from the theoretical study of potential savings, tests in order to evaluate the predictor behaviour have been made. Using the linear predictor and making 1 million simulations evaluating data inputs based on the theoretical curves and then adding Gaussian noise with a dynamic variance and amplitude (random variance and maximum value for each simulation) of up to half of the maximum of the theoretical curve, the results were as follows:

- $9,101,495$ predictions (steps for any of the thresholds)

- $8,138,433$ correct predictions

- 963,062 wrong predictions

Doing the proper calculation, an $89.42 \%$ success rate is obtained using the predictors for this specific case of simulations with these windows, commands, data variability and thresholds. It is necessary to take into account many additional factors, such as time of day, the behaviour in previous days, the time of year, the number of current users in the cell,... All these features will be incorporated in future studies, but this first approach proves that the linear predictors are a very reliable alternative to combine with the ON-OFF algorithms in the real networks.

\section{Conclusions and Further Work}

The undertaken labour exhibited in this paper has shown the great promise that the selective schemes of switching on and off base stations offer. Fixing an optimal network configuration in each of the possible cases, it is relatively easy to decide when and how increase or reduce the power emitted by the BSs, thus achieving an interesting amount of savings. Really breakthrough achievements in the field of energy savings can be obtained using these solutions, tested over live networks load profiles.

Regarding further possible work, the introduction of predictors is a step forward in the complexity of the proposal, and thanks to them a new element of evaluation appears, which is critical when making decisions about whether the ideal time to undertake the switching on or off of a BS is approaching. 
The present studies and the relative performance evaluations showed that the percentage of energy saved by applying these schemes is worthy of consideration, being necessary to validate such results in a real environment (This explains why the application of ON-OFF schemes to obtain gains in terms of power consumption in mobile networks has been selected by EARTH project as a track to be tested on a real infrastructure). To achieve the desired goals, the Telecom Italia Test Plant will be thus used in order to set up a test scenario with the necessary equipments (e.g. commercial eNB and terminals).

Acknowledgments. The work leading to this paper has received funding from the European Community's Seventh Framework Programme [FP7/2007-2013] under grant agreement $n^{\circ} 247733$ - project EARTH. This publication made by participants of the EARTH project reflects only the author's views and the European Union is not liable for any use that may be made of the information.

\section{References}

1. Mendham, S.: The implementation of AMR metering at Vodafone UK, ESTA website (February 2008)

2. Vodafone, Vodafone Group announces commitment to reduce $\mathrm{CO} 2$ emissions by $50 \%$ (April 2008) (press release)

3. Marsan, M.A., Chiaraviglio, L., Ciullo, D., Meo, M.: Optimal Energy Savings in Cellular Access Networks. In: First International Workshop on Green Communications (GreenComm 2009) (Dresden, Germany) (June 2009)

4. Oh, E., Krishnamachari, B., Liu, X., Niu, Z.: Toward Dynamic Energy-Efficient Operation of Cellular Network Infrastructure. IEEE Communications Magazine, 56-61 (2011)

5. Sánchez, L., Munoz, L.: Energy Efficiency of a Simple ON/OFF Scheme in Mobile Cellular Networks. Electronic Letters 46(20), 1404-1405 (2010)

6. EARTH project deliverable, D2.3, Energy Efficiency Analysis of the Reference Systems, Areas of Improvements and Target Breakdown (2010)

7. EARTH project deliverable, D3.1, Most Promising Tracks of Green Network Technologies (2010)

8. EARTH project deliverable, D2.2, Definition and Parameterization of Reference Systems and Scenarios (2010) 